\title{
Observations of infrared echoes around Cassiopeia A with WISE ${ }^{\star}$
}

\author{
M.-A. Besel and O. Krause
}

\author{
Max-Planck Institut für Astronomie, Königstuhl 17, 69117 Heidelberg, Germany \\ e-mail: besel@mpia.de
}

Received 21 February 2012 / Accepted 20 March 2012

\begin{abstract}
Context. Light echoes of supernovae have proven to be a viable tool for characterizing both the supernova explosion mechanism and the surrounding circumstellar and interstellar medium.

Aims. We explore new possibilities for identifying infrared echoes with WISE data and extend the area of known echoes around the supernova remnant Cassiopeia A in different illumination conditions.

Methods. Preliminary release WISE data around Cassiopeia A is analyzed and compared to Spitzer-MIPS data. While Spitzer-WISE difference images directly show evidence of infrared light echoes, they can also be identified according to their $22 / 12 \mu \mathrm{m}$ flux ratio. Results. We find that WISE allows the detection of previously unknown infrared echoes in various illumination conditions. For the first time, infrared echoes at distances greater than $800 \mathrm{pc}$ from a supernova remnant have been found. The 22/12 $\mu \mathrm{m}$ flux ratio criterion can be used as a conceptual factor for identifying infrared echoes around other supernovae as well as providing insights into the dust processing conditions of the interstellar medium.
\end{abstract}

Key words. ISM: general - dust, extinction - supernovae: individual: Cassiopeia A

\section{Introduction}

Light echoes arise from the reflection of light emitted by intense radiation events by dust in the interstellar or circumstellar medium (Chevalier 1986). At any given time, the echoing region is located on an ellipsoid whose two foci are the origin of the radiation and the observer. As the light path of the echo is longer than the direct connection between the two foci, the reflection of the radiation event can be seen after a significant time delay with respect to the initial light burst by the observer. Infrared echoes differ from their optical counterparts as we do not observe the scattered reflection, but rather the reradiated thermal emission from light absorbed by the echoing region (Wright 1980).

Scattered light echoes tend to have relatively low surface brightnesses (22-24 mag/arcsec) and may therefore be hard to find, especially when superimposed on bright field stars. This necessitates very deep high-resolution visual images, which cannot be obtained across significantly large portions of the sky, leaving blind pointings to search for echoes the only option. In contrast, the thermal radiation from infrared echoes can be very bright $(\sim 200 \mathrm{MJy} / \mathrm{sr})$ in the mid-infrared and thus has a high contrast in terms of intensity against most other sources in this wavelength regime. The Wide-Field Infrared Survey Explorer (WISE; see Wright et al. 2010), with its unprecedented complete all-sky sensitivity and resolution in the near- and mid-infrared opens new unique perspectives to systematically search for infrared echoes.

Although the first observations of light echoes were made by Wolf (1901) around Nova Persei, their full potential for Galactic astronomy was recognized only recently. Using the positional information from infrared echoes in Spitzer mid-infrared images of the supernova remnant Cas A, Krause et al. (2008a) identified the optical counterpart with a scattered light echo and were able to extract an optical spectrum of the supernova explosion.

* Table 1 is available in electronic form at http://www . aanda.org
With this spectrum, they were able to directly classify the supernova more than 330 years after the initial light flash swept past Earth. The spectrum displayed the typical features of a corecollapse type IIb supernova. Using the same technique, Krause et al. (2008b) classified Tycho Brahe's supernova as Type Ia.

Rest et al. (2011) used the spectra of optical light echoes at different scattering positions, to probe different regions of the supernova (SN) photosphere, and obtain a three-dimensional spectroscopic view of the Cas A explosion, revealing a wide range of ejecta velocities and hence probing explosion asymmetries.

Light echoes do not only contain information about the radiation event itself, but also about the material that reflects and emits the echo. In an exploratory study by Vogt et al. (2012), the dust properties of a particular bright infrared echo around Cas A were analyzed. The Spitzer-IRS spectrum reveals that the dust has been processed by the ultraviolet (UV) burst of the supernova by dust destruction and polycyclic aromatic hydrocarbon (PAH) dehydrogenation with respect to the "standard" interstellar medium (ISM). Kim et al. (2008) used multi-epoch Spitzer imaging to produce tomographic maps of the surrounding interstellar medium, revealing its three-dimensional structure. More recently, Besel et al. (in prep.) use the results of Vogt and Kim to identify the material responsible for emitting the infrared echoes: the turbulent cold and dense ISM.

\section{Observations and data reduction}

After the first discovery of rapidly moving infrared structures around Cas A by Hines et al. (2004), an extensive monitoring program was carried out in the subsequent years. With the Multiband Imaging Photometer for Spitzer (MIPS; Rieke et al. 2004 ) a roughly $3^{\circ} \times 3^{\circ}$ field was observed every year and regions with especially high activity were observed at an intermediate interval of about half a year until 2008. 

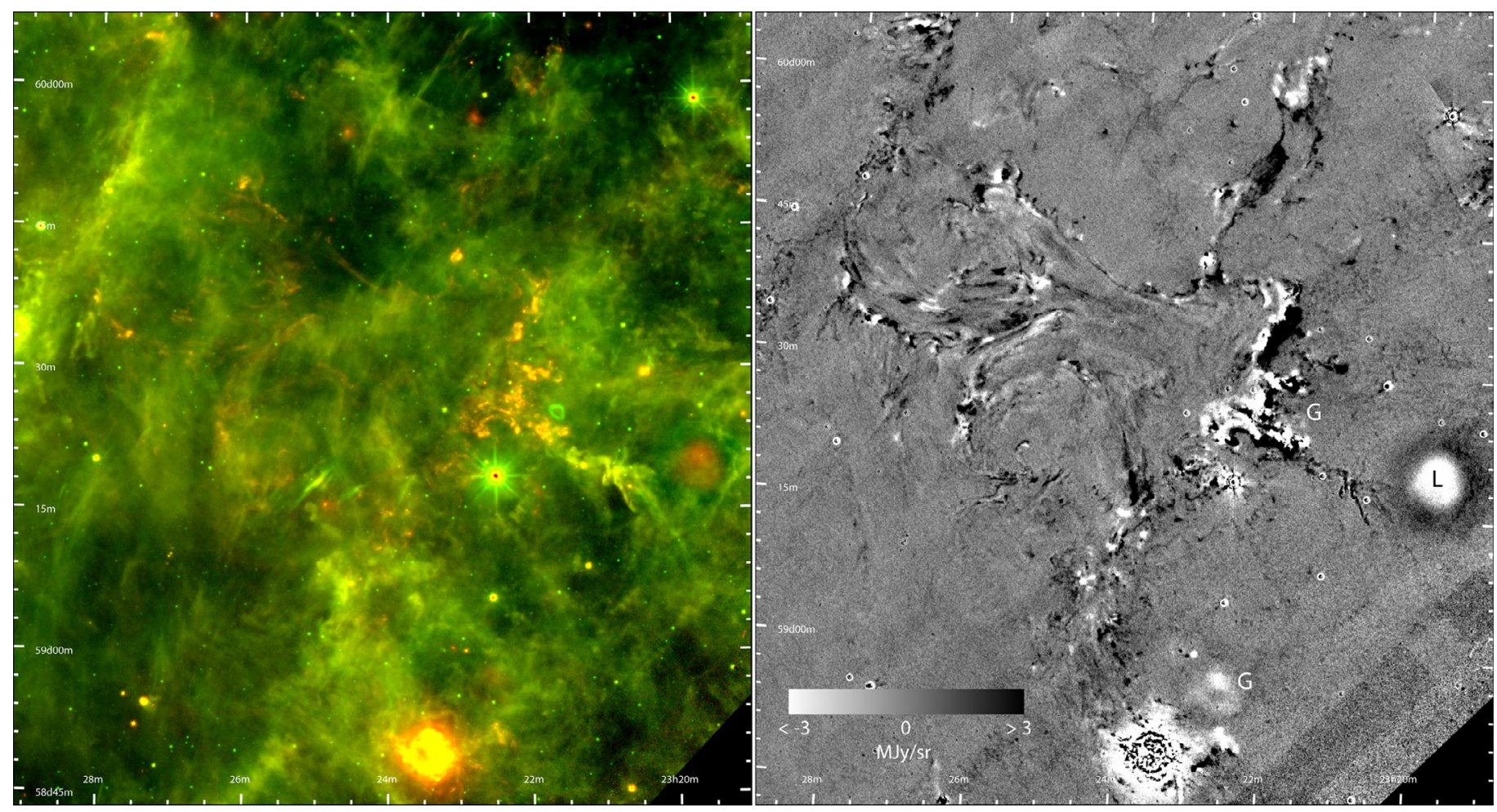

Fig. 1. Left: WISE two-color composite - red: $22 \mu \mathrm{m}$, green: $12 \mu \mathrm{m}$ - of a particular active echoing region. The Cassiopeia A supernova remnant is visible at the lower border of the image. Light echoes are clearly seen as reddish filamentary structures distributed all over the field of view. Right: two-epoch difference image from a Spitzer-MIPS $24 \mu \mathrm{m}$ (2006) image and the WISE $22 \mu \mathrm{m}$ image. Cirrus regions cancel out to a smooth background, while echoes can be identified as white (2010) or black (2006) structures. Saturated stars leave residuals owing to their complex outer PSF; a WISE latent image (marked "L") and a ghost ("G") of Cas A can be seen as white structures.

WISE started its all-sky survey on January 14 2009, at that time the spacecraft pointed its first survey leg $20^{\prime}$ to the southwest of Cas A yielding a full coverage of the eastern part of the original Spitzer IR echo monitoring area.

We obtained single exposure (level $1 \mathrm{~b}$ ) images through the NASA/IPAC Infrared Science Archive ${ }^{1}$ from an $8.5^{\circ} \times 16^{\circ}$ area adjacent to Cas A. The portion of the original monitoring area makes out approximately $1 / 20$ th of the new WISE map. Montage was used to process and co-add the single exposure images for a final pixel scale of $2.0^{\prime \prime} /$ pixel to accurately sample the instrumental point spread function (PSF; 6-12"). Zodiacal light was subtracted according to the DIRBE model as described by Kelsall et al. (1998). Median filtering of the single exposure frames was used to make basic cosmetic corrections. This removes most artifacts described in the WISE Explanatory Supplement, although not bright artifacts such as latent images or (minor-) planets. These artifacts do not impede the identification of light echoes, thus we do not try to correct for them at this time.

Most areas were covered by 10-15 frames, except for the most eastern $30^{\prime}$, where the survey coverage of WISE started. Calibration factors were applied according to the WISE Explanatory Supplement.

\section{Analysis and discussion}

We first compare WISE images to existing Spitzer-MIPS fields and then try to distinguish echo emission based on their properties in the WISE 22 and $12 \mu \mathrm{m}$ bands. With this knowledge,

\footnotetext{
${ }^{1}$ http://irsa.ipac.caltech.edu/applications/wise/
}

it is then possible to extend the area of known infrared echoes beyond the original Spitzer fields.

Since the last Spitzer maps were taken in 2008, the UV and optical bursts of the supernova illuminate different parts of interstellar clouds making the projection of echoes on the sky appear as "moving" or "varying" structures. Although echoes can appear on the same spot on the sky in different epochs, they do not probe the same position in an interstellar cloud structure. As clouds are inhomogeneous objects, it is unlikely that the same position on the sky appears exactly alike at different epochs.

Figure 1 shows a WISE two-color composite along with a Spitzer-WISE difference image. The Spitzer $24 \mu \mathrm{m}$ image (FWHM 6.45") was taken on January 292006 (PID: 20381; PI: O. Krause) and convolved to match the WISE $22 \mu \mathrm{m}$ resolution (FWHM 11.9"). Prior to subtraction, the two images were background-subtracted and scaled to the same Jy/pixel units. The resulting difference image is mostly free of signatures from cirrus clouds. Only light echoes, along with variable stars, asteroids, latent images, and other artifacts, such as those produced by saturated PSF's, can be seen. Adjacent black and white structures are indicative of "varying" or "moving" structures. Echoes in the WISE image appear as positive pixel values (white color), while the negative ones (black) belong to the Spitzer map. This method is even sensitive to very low surface brightnesses or echoes, which cannot be directly identified in raw images.

Comparing the difference image with the WISE 22$12 \mu \mathrm{m}$ color-composite reveals that infrared echoes are characterized by reddish colors with respect to surrounding cirrus clouds (green). Closer examination shows that all visually confirmed echoes have a $22 / 12 \mu \mathrm{m}$ flux ratio greater than 1.0 , which is larger than the ratio for surrounding cirrus clouds. Thus, we 

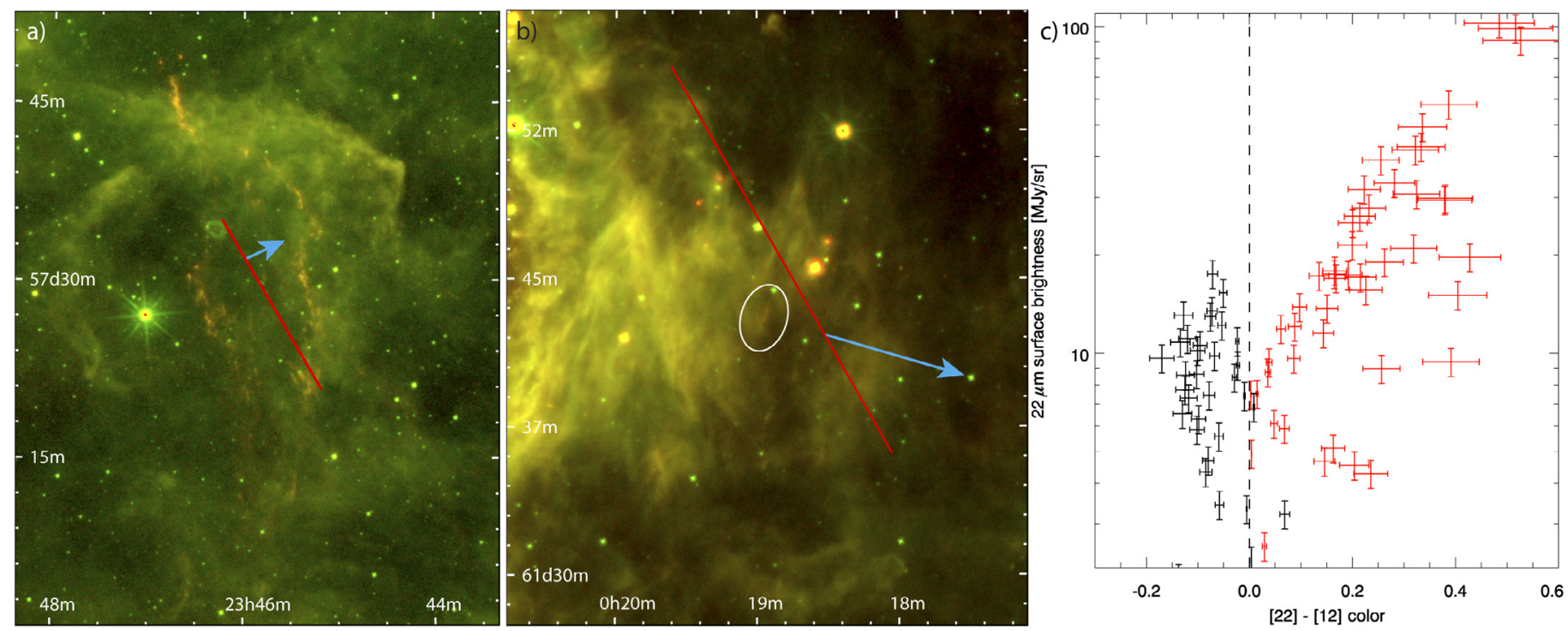

Fig. 2. a) WISE two-color composite - red: $22 \mu \mathrm{m}$, green: $12 \mu \mathrm{m}$. The red line marks the extend and position of this echoing region as previously reported in scattered light by Rest et al. (2008). This region is referenced with "O" in Table 1. b) Most distant infrared echo from Cas A at a distance $\sim 800 \mathrm{pc}$. The red line marks the location of the previously observed scattered light echo as reported in Rest et al. (2008), while the cyan arrow marks the direction towards Cas A. This region is referenced with " $\mathrm{T}$ " in Table 1. c) Color-brightness diagram of echo regions (red data points) and selected cirrus regions (black data points). The [22]-[12] color is defined as $\log _{10}\left(F_{22} / F_{12}\right)$. Errors mainly arise from uncertainties in background subtraction and flux calibration. All infrared echoes are characterized with flux ratios $>1.0$ (corresponding to a color of 0.0 ).

are able to extend the search area beyond the limit of the original $3^{\circ} \times 3^{\circ}$ maps by Spitzer, where the difference image technique cannot be applied.

Infrared echoes roughly share the same $22 / 12 \mu \mathrm{m}$ color space as young stellar objects (YSOs) or quasi stellar objects (QSOs). However, they can be discriminated in terms of their morphology. Most echoes have compact, unresolved knots, that are connected by diffuse filamentary emission, while YSOs and QSOs are isolated point-sources.

To quantify the flux ratio, we measured echo regions and selected cirrus structures across the field of view. Cirrus regions were chosen to cover the full dynamic range of observed brightnesses. Photometry was carried out relative to "floor" points separated by $\sim 17^{\circ}$ at the northern and southern edges of the field. These regions mainly contain an offset signal and feature very low cirrus emission $(\sim 1 \mathrm{MJy} / \mathrm{sr})$, as they are most distant from the Galactic plane. We performed the background subtraction as a constant offset over the entire field, as the variation in the background between the northern and southern floor points is on the order of $2 \%$, which is similar to the inter-pixel variations of $\sim 3 \%$. The main uncertainty in background subtraction arises from the remaining absolute cirrus contamination at these floor points; we estimate this uncertainty to be $7 \%$, yielding a total background subtraction uncertainty of $\sim 8 \%$.

Figure $2 \mathrm{c}$ shows a color-brightness diagram for all measured structures. Cirrus regions have an average $22 / 12 \mu \mathrm{m}$ flux ratio of $0.84 \pm 0.05$, which compares well with the expected ratio of 0.86 for the standard ISM illuminated by the standard interstellar radiation field (calculated based on dust models by Li \& Draine 2001 or Zubko et al. 2004, in concordance with DIRBE and IRAS observations). All light echoes are characterized by $22 / 12 \mu \mathrm{m}$ flux ratios greater than $\sim 1.0$. As the local cirrus structure varies on the same spatial scales as light echoes, it is impossible to calculate a physical $22 / 12 \mu \mathrm{m}$ ratio that is intrinsic to the echo region itself. Estimation of the local background is especially difficult at $12 \mu \mathrm{m}$, where the cirrus-echo contrast is at its lowest. Any estimation would be unable to derive reliable flux ratios. For this reason, we cannot comment on the possibility of varying flux ratios in different echo regions at this time.

However, we point out that data acquired at a second observing epoch could help us to precisely disentangle echo emission from cirrus emission and would yield interesting insights into dust processing by the supernova radiation. The $12 \mu \mathrm{m}$ band is very sensitive to the $11.3 \mu \mathrm{m}$ PAH C-H out-of-plane bending mode, which was found to be highly suppressed by Vogt et al. (2012); large $22 / 12 \mu \mathrm{m}$ ratios are indicative of both dust destruction and PAH dehydrogenation. As the radiation field diminishes as $r^{-2}$, one would expect the ratio to decrease with distance from the supernova. At a distance of $d=60 \mathrm{pc}$ from the supernova, the expected flux ratios for infrared light echoes are 2.5 for processed and 1.4 for pristine dust. These ratios change to 1.7 and 1.0 at a distance of $d=600 \mathrm{pc}$.

Flux ratio estimates based on global background subtraction are given in Table 1 in the online material. As these values still include local cirrus contamination, the intrinsic values may be higher by several tens of percent. The resulting ratios are within the expected range for both processed and pristine dust.

Figure 3 sketches the locations of all infrared echoing regions from aforementioned WISE field. The outer regions represent the first observations of infrared echoes at distances $\gtrsim 150 \mathrm{pc}$. Full positional properties are also given in Table 1 .

\section{Outlook}

WISE allows the detection of previously unknown infrared echoes around Cas A. The final data release will also include echo regions on the western side of the remnant seen on Spitzer images, but not covered in the preliminary release data.

The complete mapping of infrared echoes allows new pointed searches for scattered light echoes, and follow-up spectroscopy of the scattered supernova spectrum under new illumination and scattering conditions, possibly revealing new knowledge about supernova explosion mechanisms and asymmetries. 


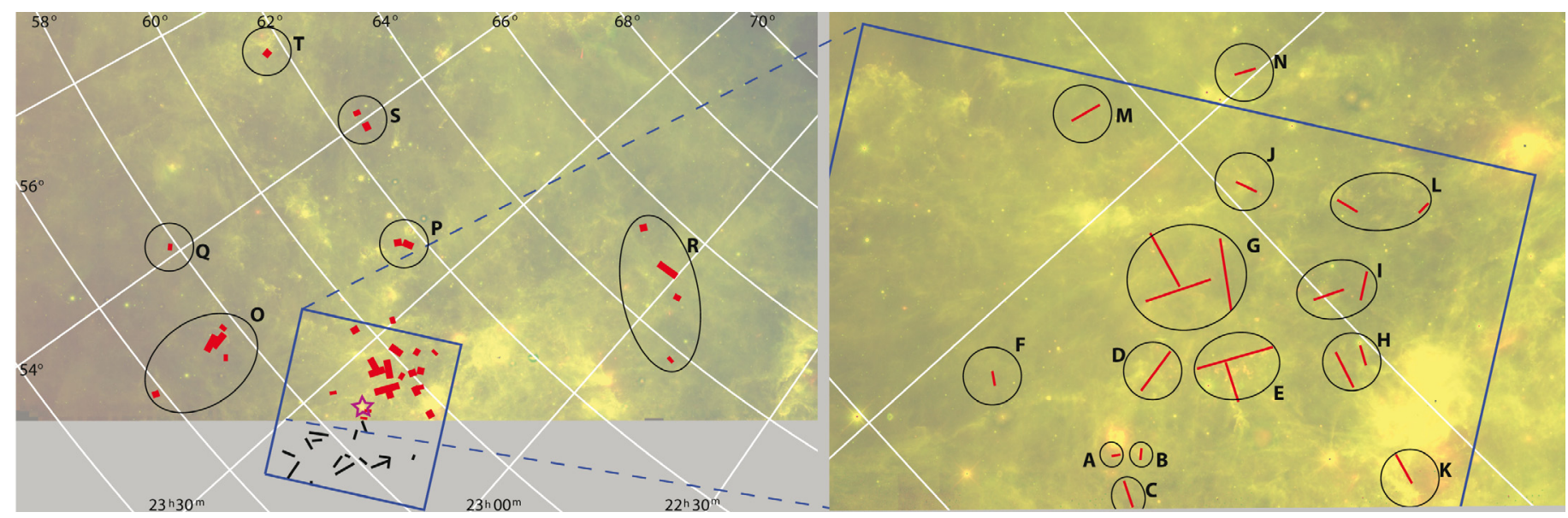

Fig. 3. Sketch of echo positions (red markers) in the field described in this study. The original $3^{\circ} \times 3^{\circ}$ MIPS field is depicted in blue with the major light echo positions in black (for clarity only MIPS-LE positions outside the WISE coverage are marked). The full positions and properties of marked regions are presented in Table 1 in the online material.

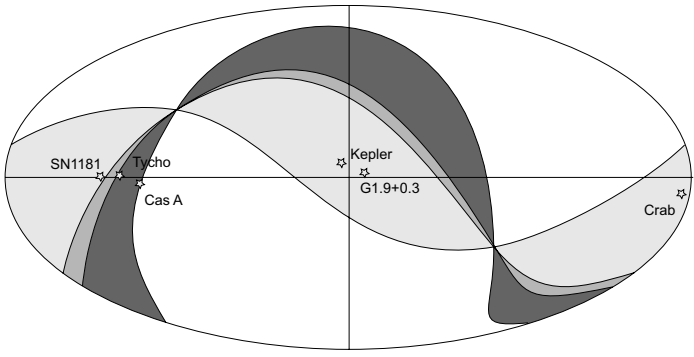

Fig. 4. Dual-epoch coverage in a Galactic coordinate illustration. The colored areas have been observed during a second epoch with a time gap of approximately six months under cryogenic conditions. The darkest shade corresponds to the area covered by all photometric bands. The $22 \mu \mathrm{m}$ band was disabled in the middle shade, and both $22 \mu \mathrm{m}$ and $12 \mu \mathrm{m}$ were disabled in the lightest area. Although the white areas are also covered in a second epoch of observations, only the two shortest wavebands were used. Data from this region were used exclusively by the NEOWISE mission extension and will not be published in imaging formats.

As the spacecraft started its survey close to the supernova remnant (SNR), the aforementioned area was observed for a second time after the first all-sky scan was completed on July 17 2010. WISE continued survey observations until February 92011 allowing a second (and partially third) all-sky scan. However, owing to the cryogen exhaustion on September 29 2010, not all photometric bands were used for the entire time. The lowest coverage is found in the $22 \mu \mathrm{m}$ band, but still includes the entire area west of Cas A described here. At $12 \mu \mathrm{m}$, the dual-epoch coverage is larger (10 degrees, at the ecliptic equator) and includes an area with known optical light echoes around Tycho's supernova remnant. The largest cryogenic coverage is found in the two shortest wavelength bands and includes areas around the historical supernova remnants SN1181, Kepler, Crab, and G1.9+0.3 (see Fig. 4). However, these short wavelengths are already very crowded by the stellar emission from field stars. As WISE has a very stable PSF, the dual-epoch difference image should nevertheless reveal bright infrared echoes from these supernovae.

This second epoch data would also enable us to perform an accurate determination of the superimposed cirrus component, yielding physical 22/12 $\mu \mathrm{m}$ flux ratios for echo regions that changed their position sufficiently during these two epochs. Such studies will help to provide new insights into ISM structure and dust physics. Dust destruction efficiencies can be mapped as a function of distance from the supernova; in turn, a change in the dust destruction efficiencies can map supernova-explosion, circumstellar-material and interstellar-material properties.

Acknowledgements. This publication makes use of data products from the Wide-Field Infrared Survey Explorer, which is a joint project of the University of California, Los Angeles, and the Jet Propulsion Laboratory/California Institute of Technology, funded by the National Aeronautics and Space Administration. This research also made use of Montage, funded by the National Aeronautics and Space Administration's Earth Science Technology Office, Computation Technologies Project, under Cooperative Agreement Number NCC5-626 between NASA and the California Institute of Technology. Montage is maintained by the NASA/IPAC Infrared Science Archive.

\section{References}

Chevalier, R. A. 1986, ApJ, 308, 225

Hines, D. C., Rieke, G. H., Gordon, K. D., et al. 2004, ApJS, 154, 290

Kelsall, T., Weiland, J. L., Franz, B. A., et al. 1998, ApJ, 508, 44

Kim, Y., Rieke, G. H., Krause, O., et al. 2008, ApJ, 678, 287

Krause, O., Birkmann, S. M., Usuda, T., et al. 2008a, Science, 320, 1195

Krause, O., Tanaka, M., Usuda, T., et al. 2008b, Nature, 456, 617

Li, A., \& Draine, B. T. 2001, ApJ, 554, 778

Rest, A., Welch, D. L., Suntzeff, N. B., et al. 2008, ApJ, 681, L81

Rest, A., Foley, R. J., Sinnott, B., et al. 2011, ApJ, 732, 3

Rieke, G. H., Young, E. T., Engelbracht, C. W., et al. 2004, ApJS, 154, 25

Vogt, F. P. A., Besel, M.-A., Krause, O., \& Dullemond, C. P. 2012, ApJ, accepted [arXiv: 1203.1646]

Wolf, M. 1901, Astron. Nachr., 157, 161

Wright, E. L. 1980, ApJ, 242, L23

Wright, E. L., Eisenhardt, P. R. M., Mainzer, A. K., et al. 2010, AJ, 140, 1868

Zubko, V., Dwek, E., \& Arendt, R. G. 2004, ApJS, 152, 211 
M.-A. Besel and O. Krause: Infrared echoes around Cas A with WISE

Table 1. Positions and properties of detected infrared light echoes around Cassiopeia A.

\begin{tabular}{|c|c|c|c|c|c|c|c|c|}
\hline $\begin{array}{l}\text { Region } \\
\text { identifier }\end{array}$ & $\begin{array}{c}\text { RA } \\
\text { J2000 }\end{array}$ & $\begin{array}{c}\text { Dec } \\
\text { J2000 }\end{array}$ & $\begin{array}{l}\text { Distance } \\
{[\mathrm{pc}]} \\
(1)\end{array}$ & $\begin{array}{c}z \\
{[\mathrm{pc}]} \\
(2)\end{array}$ & $\begin{array}{l}\text { Scattering angle } \\
\text { [degrees] } \\
\text { (3) }\end{array}$ & $\begin{array}{c}\text { PA } \\
\text { [degrees] } \\
(4)\end{array}$ & $\begin{array}{c}\text { MSB @ } 22 \mu \mathrm{m} \\
{[\mathrm{MJy} / \mathrm{sr}]} \\
(5)\end{array}$ & $\begin{array}{c}22 \mu \mathrm{m} / 12 \mu \mathrm{m} \\
\text { ratio } \\
\text { (6) }\end{array}$ \\
\hline $\bar{A}$ & $23^{\mathrm{h}} 23^{\mathrm{m}} 6^{\mathrm{s}}$ & $58^{\circ} 45^{\mathrm{m}} 15^{\mathrm{s}}$ & 52.1 & -51.9 & 175.1 & -141.8 & 17.89 & 2.43 \\
\hline B & $23^{\mathrm{h}} 22^{\mathrm{m}} 31^{\mathrm{s}}$ & $58^{\circ} 52^{\mathrm{m}} 1^{\mathrm{s}}$ & 52.3 & -51.7 & 171.3 & -66.3 & 21.87 & 1.64 \\
\hline $\mathrm{C}$ & $23^{\mathrm{h}} 21^{\mathrm{m}} 16^{\mathrm{s}}$ & $58^{\circ} 38^{\mathrm{m}} 38^{\mathrm{s}}$ & 53.9 & -50.0 & 158.5 & -120.5 & 25.83 & 2.08 \\
\hline D & $23^{\mathrm{h}} 23^{\mathrm{m}} 42^{\mathrm{s}}$ & $59^{\circ} 12^{\mathrm{m}} 21^{\mathrm{s}}$ & 54.6 & -49.2 & 154.7 & 4.5 & 23.35 & 1.71 \\
\hline $\mathrm{E}$ & $23^{\mathrm{h}} 22^{\mathrm{m}} 12^{\mathrm{s}}$ & $59^{\circ} 23^{\mathrm{m}} 16^{\mathrm{s}}$ & 58.1 & -45.7 & 142.4 & -15.7 & 95.01 & 2.68 \\
\hline $\mathrm{F}$ & $23^{\mathrm{h}} 28^{\mathrm{m}} 18^{\mathrm{s}}$ & $58^{\circ} 35^{\mathrm{m}} 49^{\mathrm{s}}$ & 59.5 & -44.2 & 138.6 & 108.6 & 3.31 & 1.05 \\
\hline G & $23^{\mathrm{h}} 26^{\mathrm{m}} 3^{\mathrm{s}}$ & $59^{\circ} 48^{\mathrm{m}} 59^{\mathrm{s}}$ & 71.1 & -32.3 & 118.1 & 17.9 & 35.19 & 1.88 \\
\hline $\mathrm{H}$ & $23^{\mathrm{h}} 19^{\mathrm{m}} 1^{\mathrm{s}}$ & $59^{\circ} 48^{\mathrm{m}} 36^{\mathrm{s}}$ & 74.5 & -28.8 & 113.9 & -29.2 & 15.12 & 1.61 \\
\hline I & $23^{\mathrm{h}} 21^{\mathrm{m}} 34^{\mathrm{s}}$ & $60^{\circ} 3^{\mathrm{m}} 35^{\mathrm{s}}$ & 79.5 & -23.6 & 108.5 & -10.8 & 15.06 & 1.56 \\
\hline $\mathrm{J}$ & $23^{\mathrm{h}} 27^{\mathrm{m}} 23^{\mathrm{s}}$ & $60^{\circ} 4^{\mathrm{m}} 57^{\mathrm{s}}$ & 83.7 & -19.3 & 104.7 & 21.0 & 27.45 & 1.87 \\
\hline K & $23^{\mathrm{h}} 14^{\mathrm{m}} 37^{\mathrm{s}}$ & $59^{\circ} 40^{\mathrm{m}} 26^{\mathrm{s}}$ & 86.4 & -16.5 & 102.4 & -51.8 & 37.89 & 2.45 \\
\hline $\mathrm{L}$ & $23^{\mathrm{h}} 23^{\mathrm{m}} 51^{\mathrm{s}}$ & $60^{\circ} 22^{\mathrm{m}} 58^{\mathrm{s}}$ & 93.9 & -8.8 & 96.9 & 1.8 & 7.46 & 1.43 \\
\hline M & $23^{\mathrm{h}} 33^{\mathrm{m}} 20^{\mathrm{s}}$ & $59^{\circ} 45^{\mathrm{m}} 4^{\mathrm{s}}$ & 93.9 & -8.8 & 96.9 & 52.3 & 8.06 & 1.25 \\
\hline $\mathrm{N}$ & $23^{\mathrm{h}} 30^{\mathrm{m}} 25^{\mathrm{s}}$ & $60^{\circ} 25^{\mathrm{m}} 54^{\mathrm{s}}$ & 109.3 & 7.1 & 88.1 & 27.7 & 8.66 & 1.28 \\
\hline $\mathrm{O}$ & $23^{\mathrm{h}} 46^{\mathrm{m}} 43^{\mathrm{s}}$ & $57^{\circ} 45^{\mathrm{m}} 4^{\mathrm{s}}$ & 223.1 & 124.4 & 59.3 & 106.7 & 17.71 & 2.11 \\
\hline $\mathrm{P}$ & $23^{\mathrm{h}} 37^{\mathrm{m}} 55^{\mathrm{s}}$ & $61^{\circ} 43^{\mathrm{m}} 49^{\mathrm{s}}$ & 242.5 & 144.3 & 56.9 & 30.0 & 11.74 & 1.45 \\
\hline Q & $0^{\mathrm{h}} 1^{\mathrm{m}} 31^{\mathrm{s}}$ & $57^{\circ} 50^{\mathrm{m}} 25^{\mathrm{s}}$ & 445.9 & 354.0 & 42.5 & 97.0 & 4.12 & 1.54 \\
\hline $\mathrm{R}$ & $23^{\mathrm{h}} 1^{\mathrm{m}} 43^{\mathrm{s}}$ & $65^{\circ} 10^{\mathrm{m}} 21^{\mathrm{s}}$ & 705.5 & 621.6 & 35.1 & -19.5 & 3.77 & 1.15 \\
\hline S & $0^{\mathrm{h}} 1^{\mathrm{m}} 44^{\mathrm{s}}$ & $62^{\circ} 30^{\mathrm{m}} 54^{\mathrm{s}}$ & 571.5 & 483.4 & 38.2 & 47.6 & 3.19 & 1.13 \\
\hline $\mathrm{T}$ & $0^{\mathrm{h}} 18^{\mathrm{m}} 57^{\mathrm{s}}$ & $61^{\circ} 42^{\mathrm{m}} 56^{\mathrm{s}}$ & 799.4 & 718.4 & 33.5 & 61.2 & 4.95 & 1.13 \\
\hline
\end{tabular}

Notes. The table lists the properties of the brightest echoing features in the regions defined in the Fig. 3. (1) Direct distance between supernova and echoing dust cloud. (2) Distance between supernova and dust cloud along the line of sight - negative values indicate a position behind the SNR with respect to Earth. (3) Scattering angle. (4) Position angle with respect to the SNR. North is $0^{\circ}$, east $+90^{\circ}$. (5) Maximum surface brightness in the $22 \mu \mathrm{m}$ band; no local cirrus subtraction. (6) $22 / 12 \mu \mathrm{m}$ flux ratio average across the region; no local cirrus subtraction. 